(c) 2019 IEEE Personal use of this material is permitted. Permission from IEEE must be obtained for all other uses, in any current or future media, including reprinting/republishing this material for advertising or promotional purposes, creating new collective works, for resale or redistribution to servers or lists, or reuse of any copyrighted component of this work in other works. 


\section{CARESSES:The Flower that Taught Robots about Culture}

\author{
Antonio Sgorbissa \\ University of Genova, Genova, Italy \\ antonio.sgorbissa@unige.it \\ Alessandro Saffiotti \\ Örebro University, Örebro, Sweden \\ asaffio@aass.oru.se \\ Nak Young Chong \\ JAIST, Kanazawa, Japan \\ nakyoung@jaist.ac.jp \\ Linda Battistuzzi \& Roberto Menicatti \\ University of Genova, Genova, Italy \\ linda.battistuzzi@unige.it \\ roberto.menicatti@dibris.unige.it \\ Federico Pecora \\ Örebro University, Örebro, Sweden \\ fpa@aass.oru.se
}

\author{
Irena Papadopoulos \\ Middlesex University, London, UK \\ R.Papadopoulos@mdx.ac.uk \\ Amit Kumar Pandey \\ SoftBank Robotics Europe, Paris, France \\ akpandey@softbankrobotics.com \\ Hiroko Kamide \\ Nagoya University, Nagoya, Japan \\ kamide@coi.nagoya-u.ac.jp \\ Christina Koulouglioti \\ Middlesex University, London, UK \\ C.Koulouglioti@mdx.ac.uk \\ Sanjeev Kanoria \\ Advinia HelthCare, London, UK \\ sanjkan@gmail.com \\ Raffaele Mastrolonardo \\ Effecinque, Genova, Italy \\ raffaele@effecinque.org
}

\author{
Chris Papadopoulos \\ University of Bedfordshire, Luton, UK \\ Chris.Papadopoulos@beds.ac.uk \\ Len Merton \\ Advinia HelthCare, London, UK \\ ceo@advinia.com \\ Jaeryoung Lee \\ Chubu University, Kasugai, Japan \\ jaeryoung.lee@gmail.com \\ Gurch Randhawa \\ University of Bedfordshire. Luton, UK \\ Gurch.Randhawa@beds.ac.uk \\ Yuto Lim \\ JAIST, Kanazawa, Japan \\ ylim@jaist.ac.jp
}

\begin{abstract}
The video describes the novel concept of "culturally competent robotics", which is the main focus of the project CARESSES (Culturally-Aware Robots and Environmental Sensor Systems for Elderly Support). CARESSES a multidisciplinary project whose goal is to design the first socially assistive robots that can adapt to the culture of the older people they are taking care of. Socially assistive robots are required to help the users in many ways including reminding them to take their medication, encouraging them to keep active, helping them keep in touch with family and friends. The video describes a new generation of robots that will perform their actions with attention to the older person's customs, cultural practices and individual preferences.
\end{abstract}

Keywords-Culturally competent robots; elderly care.

\section{DESCRIPTION OF THE VIDEO}

The video ${ }^{1}$ describes the ground breaking objective of building culturally competent robots, able to operate in a range of settings from an individual's home to a care facility.

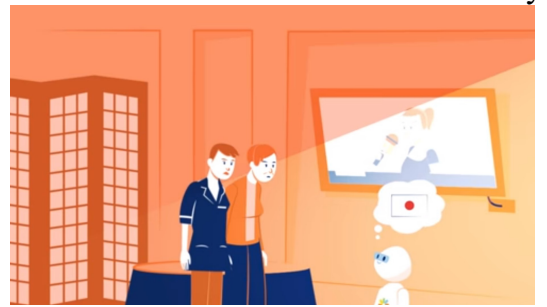

Culturally competent robots are able to autonomously reconfigure their way of acting and speaking, when offering a service, to match the culture, customs, and preferences of the person they are assisting. The need for cultural competence (grounded in cultural-awareness, cultural knowledge and cultural sensitivity) in healthcare has been widely investigated in the nursing literature. The study of Transcultural Nursing

\footnotetext{
${ }^{1}$ The video is not archived in the HRI 2019 Proceedings since the authors are not authorized to transfer the copyright of the video. See https://youtu.be/eLTKhfVYrTw
}

and Cultural Competence plays a crucial role in providing culturally appropriate nursing care, and it is supported by worldwide associations ${ }^{2}$.

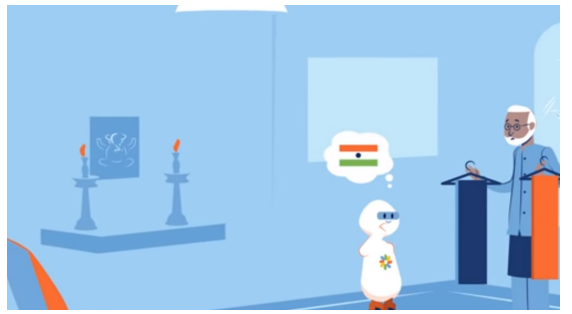

The video suggests that making cultural competent systems is key to address one of the major problems in assistive robotics: how to increase acceptability by being more sensitive to the user's needs, customs and lifestyle, thus producing a greater impact on the quality of life of users and their caregivers, reducing caregiver burden, and improving the system's efficiency and effectiveness. From the commercial perspective, cultural competence is crucial to overcome the barriers to marketing robots across different countries, and can pave the way to the use of socially assistive robots in a number of different domains, from education to tourism.

\section{ACKNOWLEDGEMENTS}

CARESSES $^{3}$ has received funding of EUR 2,084,248.75 from the European Union's Horizon 2020 research and innovation programme under grant agreement No 737858, and JPY $60,000,000$ from the Ministry of Internal Affairs and Communications of Japan.

[1] B. Bruno, N.Y. Chong, H. Kamide, S. Kanoria, J. Lee, Y. Lim, A. K. Pandey, C. Papadopoulos, I. Papadopoulos, F. Pecora, A. Saffiotti, A. Sgorbissa, 6th IEEE International Symposium on Robot and Human Interactive Communication (RO-MAN), Lisbon, 201

\footnotetext{
${ }^{2}$ http://europeantransculturalnurses.eu/

${ }^{3}$ www.caressesrobot.org
} 